months, the mean number of consultations with HCPs increased with disease severity (3.7 mild, 4.2 moderate and 5.7 severe $[<0.001])$. This pattern was also observed in relation to the mean number of tests/scans conducted in the last 12 months $(6.9$ mild, 7.9 moderate and 9.3 severe $[<0.001])$. More than a quarter of severe patients visited the ER in the last 12 months (26\% vs. $4 \%$ mild; $9 \%$ moderate $[<0.001]$ ) and visits to hospital increased with disease severity (Table 1). The proportion of patients that have had a surgery due to their OA rose with worsening disease severity $(11 \%, 13 \%$ and $27 \%$ for mild, moderate and severe, respectively $[<0.001])$.

Table 1. Physician-reported healthcare burden by OA disease severity

\begin{tabular}{lccc}
\hline & $\begin{array}{c}\text { Mild } \\
(\mathrm{n}=874)\end{array}$ & $\begin{array}{c}\text { Moderate } \\
(\mathrm{n}=1904)\end{array}$ & $\begin{array}{c}\text { Severe } \\
(\mathrm{n}=818)\end{array}$ \\
\hline $\begin{array}{c}\text { Number of patient visits to ER in the last } 12 \text { months, } \\
\text { mean (SD) }\end{array}$ & $0.1(0.4)$ & $0.1(0.6)$ & $0.5(1.0)$ \\
$\begin{array}{c}\text { Patients with } \geq 1 \text { emergency visit in the last } 12 \\
\text { months, } \mathrm{n}(\%)\end{array}$ & $13(1.5)$ & $43(2.3)$ & $79(9.7)$ \\
$\begin{array}{c}\text { Patients with } \geq 1 \text { hospitalisation in the last } 12 \text { months, } \\
\mathrm{n}(\%)\end{array}$ & $11(0.1)$ & $9(0.5)$ & $26(3.2)$ \\
$\begin{array}{c}\text { Number of patient outpatient hospital visits in the last } \\
12 \text { months, mean (SD) }\end{array}$ & $0.5(1.4)$ & $0.6(1.1)$ & $1.2(1.4)$ \\
\hline
\end{tabular}

Conclusion: This real-world data demonstrated an increase in visits to HCPs, monitoring tests and scans, hospitalisations, ER visits and surgery as OA disease severity worsened.

Disclosure of Interests: Philip G Conaghan Consultant of: AbbVie, BMS, Eli Lilly, EMD Serono, Flexion Therapeutics, Galapagos, GSK, Novartis, Pfizer, Speakers bureau: AbbVie, Eli Lilly, Novartis, Pfizer, Lucy Abraham Shareholder of: Pfizer, Employee of: Pfizer, Peita Graham-Clarke Shareholder of: Eli Lilly and Co, Employee of: Eli Lilly and Co, Lars Viktrup Shareholder of: Eli Lilly and Company, Employee of: Eli Lilly and Company, Joseph C Cappelleri Shareholder of: Pfizer Inc, Employee of: Pfizer Inc, Craig Beck Shareholder of: Pfizer, Employee of: Pfizer, Andrew G Bushmakin Shareholder of: Pfizer Inc, Employee of: Pfizer Inc, Niall Hatchell: None declared, Emily Clayton: None declared, James Jackson: None declared

DOI: 10.1136/annrheumdis-2020-eular.5513

\begin{tabular}{|l|l}
\hline FRI0398 & COMPARISON OF THE EFFICACY AND SAFETY OF \\
TWO HYALURONIC ACIDS IN THE TREATMENT OF \\
KNEE OSTEOARTHRITIS
\end{tabular}

B. Cortet ${ }^{1}, \mathrm{~S}$. Lombion ${ }^{2}$, O. Bruyere ${ }^{3} .{ }^{1}$ Department of Rheumatology and UR 4490, University-Hospital of Lille, Lille, France; ${ }^{2}$ SLC Consulting, Geneva, Switzerland; ${ }^{3}$ WHO Collaborating Centre for Public Health Aspects of MusculoSkeletal Health and Ageing \& Division of Public Health, Epidemiology and Health Economics, University of Liège, Liege, Belgium

Background: Several viscosupplement treatments are available to patients suffering from osteoarthritis (OA) but few comparative clinical trials have been conducted.

Objectives: The primary objective of the study was to demonstrate at 24 weeks the non-inferiority of on hyaluronic acid over a second one in terms of efficacy (pain relief) in knee OA patients (Kellgren and Lawrence radiologic stage II or III) with whom oral treatment had failed.

Methods: This was a prospective, multicenter, comparative, randomized, double-blinded study (one independent physician evaluator-one physician injector), comparing two viscosupplements: one containing a solution of hyaluronic acid (SYNOLIS VA $® 80 \mathrm{mg}$ hyaluronic acid and $160 \mathrm{mg}$ sorbitol - Group HA1) and the other containing one of Hylan (SYNVISC ONE $® 48 \mathrm{mg}$ Hylane GF-20 - Group HA2) over a period of 24 weeks. At inclusion, the average VAS Pain (1-100) was 62.5. The patients were randomized in 2 parallel groups at DO and followed until D168. They received an injection of either HA1 or HA2. Efficacy was primarily assessed using the WOMAC Pain index (daily assessed by the patient during seven days following the injection, and then at D14). During the follow up visits (D28-D84-D168) WOMAC pain, stiffness and function scores were assessed as secondary objectives. At D168, efficacy and satisfaction were also evaluated by the evaluator and by the patient using Likert scale ( 7 points). Moreover, the number of responders strict each group was evaluated according to the OMERACT-OARSI criteria. According to methodology guidelines, the per protocol (PP) population has been used as primary analysis. The PP population included all patients from the intention to treat (ITT) population who completed the study without any major protocol violation.
Results: 202 patients were randomized (ITT population, 96 in the HA1 group and 106 in the HA2 group). Baseline demographic data for the PP population at the time of randomization by treatment group. Patients were predominantly female $(66 \%)$. The median age of the whole population was 65 years and the median body mass index of $27.4 \mathrm{~kg} / \mathrm{m}^{2}$. No statistically significant differences between the two treatment groups were observed for any demographic criteria. At D168, 197 presented no protocol violations (94 in the HA1 group and 103 in the HA2 group). This population had a decrease on the overall score of the WOMAC Pain at -29.2+/- 24.1 (SD) in the HA1 group and -31.6 +/-25.5 (SD) in the HA2 group confirming the non-inferiority $(P=0.57$ for the difference between groups). Regarding the secondary endpoints, no significant difference has been observed at D14, D28, D84, D168, in the PP population for all the outcome except stiffness at D28. There was also no difference between the responders rate in two groups ( $79 \%$ for HA1 and $77 \%$ for HA2). In terms of safety, both products were well tolerated. No case of allergy or infection in the course of the injection was reported. Serious adverse events have been reported by 4 patients in HA1 group and 3 in HA2 group.

Conclusion: In this study, we confirmed the non-inferiority of HA1 compared with HA2 in terms of both efficacy and safety.

Disclosure of Interests: Bernard Cortet Consultant of: Aptissen, Sandrine Lombion Consultant of: Aptissen, Olivier Bruyere Consultant of: Aptissen DOI: 10.1136/annrheumdis-2020-eular.5617

\section{FRI0399 COLCHICINE IS NOT EFFECTIVE FOR REDUCING OSTEOARTHRITIC HAND PAIN COMPARED TO PLACEBO: A RANDOMISED, PLACEBO-CONTROLLED TRIAL (COLAH)}

C. Davis ${ }^{1,2}$, C. Ruediger ${ }^{1,2}$, K. Dyer ${ }^{2}$, S. Lester ${ }^{1,2}$, S. Graf ${ }^{3}$, F. P. B. Kroon ${ }^{4}$, S. Whittle ${ }^{2}$, C. Hill ${ }^{1,2}{ }^{1}$ University of Adelaide, Discipline of Medicine, Adelaide, Australia; ${ }^{2}$ Queen Elizabeth Hospital, Rheumatology Department, Woodville South, Australia; ${ }^{3}$ Wakefield House Rheumatology, Adelaide, Australia; ${ }^{4}$ Leiden University Medical Center, Department of Rheumatology, Leiden, Netherlands

Background: Current pharmacotherapies to treat or prevent hand osteoarthritis are limited. Colchicine, an anti-inflammatory agent effective at reducing joint pain and swelling in gouty arthritis, may offer relief in hand osteoarthritis, though this has not been investigated before.

Objectives: To investigate the efficacy of colchicine compared to placebo on VAS pain scores over 12 weeks in adults with hand osteoarthritis in a randomised, double-blind controlled trial.

Methods: 64 community-dwelling adults with hand osteoarthritis (American College of Rheumatology criteria) ${ }^{2}$ (54 females, 48-79 years) were randomised $1: 1$ to colchicine $(0.5 \mathrm{mg}$ twice daily) or placebo for 12 weeks. VAS pain scores (worst affected hand) were obtained at baseline and weeks 6 , 12 , and after treatment withdrawal at week 16 . Secondary outcome measures included grip strength, $\mathrm{C}$-reactive protein (CRP) and tender and swollen joint count (TSJC). Grip strength, TSJC and CRP were obtained at baseline and week 12. Intention-to-treat analyses, adjusted for age and gender, were performed using constrained longitudinal data analysis models in Stata v16. ${ }^{3}$ This study is registered with the Australia New Zealand Clinical Trials Registry, ACTRN12617001524381.

Results: 58 participants completed the study ( $\mathrm{N}=27$ colchicine, $\mathrm{N}=31$ placebo, withdrawal rate $9 \%$ ). Mean (S.D) VAS score of the affected hand at baseline was $71.4(14.5) \mathrm{mm}$ in the placebo and $65.4(15.0) \mathrm{mm}$ in the colchicine group $(\mathrm{p}$ $=0.11$ ). VAS scores improved during treatment, but were comparable between groups at week 6, 12 and 16 (Table 1). There were no differences between groups at week 12 for CRP, TSJC or grip strength (Table 1). Adverse events related to study medications included nausea $(n=4)$, diarrhoea $(n=9)$, vomiting $(n=3)$, bloating $(n=1)$ and reflux $(n=1)$.

Table 1. COLAH study primary and secondary outcomes, from constrained longitudinal data analysis model

\begin{tabular}{llcccc}
\hline Outcome & Timepoint & Colchicine (SE) & Placebo (SE) & $\begin{array}{c}\text { Colchicine-Placebo } \\
(95 \% \mathrm{Cl})\end{array}$ & p-value \\
& & & & & \\
\hline VAS Pain (mm) & 6 weeks & $53.5(4.5)$ & $53.9(4.6)$ & $-0.4(-12.3,13.1)$ & 0.95 \\
& 12 weeks & $57.1(4.4)$ & $48.4(4.6)$ & $8.7(-21.2,3.9)$ & 0.18 \\
& 16 weeks & $62.0(4.3)$ & $61.6(3.7)$ & $-0.4(-11.4,10.5)$ & 0.94 \\
TSJC (0-20) & 12 weeks & $5.6(0.7)$ & $3.8(0.7)$ & $1.8(-4.1,0.5)$ & 0.13 \\
Grip strength (kg) & 12 weeks & $14.4(0.8)$ & $15.3(0.8)$ & $-0.9(-0.2,2.0)$ & 0.10 \\
CRP (mg/L) & 12 weeks & $4.5(1.4)$ & $4.0(1.3)$ & $0.5(-3.8,2.9)$ & 0.77
\end{tabular}

